\title{
Gemeinsam lesen?
}

Von: Julika Griem

Zurzeit trösten wir uns auch am KWI manchmal damit, gemeinsam einsam zu sein, um in der Pandemie möglichst geduldig zu bleiben. In unserer Forschungsarbeit - und seit genau einem Jahr auch auf diesem Blog - denken wir aber auch darüber nach, wie wir nicht nur als Einzeltäter*innen forschen, schreiben und lesen. In philologischen und kulturwissenschaftlichen Kontexten wurde dies immer schon getan, wenn auch nicht ausreichend reflektiert. Stärker als über Sammelbände, gemeinsam geschriebene Aufsätze und Anträge oder gar Manifeste definieren wir uns über individualisierte Formate und subjektivierte Produktionsweisen, und über die Fiktion eines Personalstils. Auch wenn uns bewusst ist, dass wir durch Nachahmung lernen und Jargon nicht immer widerstehen, unter Zeitdruck Formulierungsroutinen übernehmen oder Textbausteine zuliefern lassen. Neben einem wachsenden Interesse für implizite, halbbewusste oder gar verdrängte Formen geisteswissenschaftlicher Kollaboration entstehen gegenwärtig zudem neue Formen und Foren, in denen gezielter erprobt wird, wie wir in unseren Fächern sinnvoll gemeinsam agieren können. Digitale Werkzeuge erleichtern die kooperative Texterstellung, und auch Tendenzen des „social“ und „shared reading“ zeugen von einer geschärften Aufmerksamkeit für Praktiken, Techniken und Stile kollektiver und kooperativer Autor*innenschaft, Informationsverarbeitung und Wissensproduktion.

Ein wichtiger Faktor in der Geschichte des Lesens geschriebener und gedruckter Texte ist die Intimität von Lektüren. So ist es möglich, in einer vollbesetzten U-Bahn mit Körperkontakt zu seinen Nachbarn zu sitzen und sich dennoch zurückzuziehen. Hier lesen Menschen nebeneinander, aber noch nicht gemeinsam, denn es wird ja keine Leseerfahrung geteilt. Seit mehr als zwei Jahrzehnten hat sich insbesondere in den USA, Kanada und Großbritannien das Interesse an teilbaren und gemeinschaftsstiftenden Lese-Formaten verstärkt. Unter dem Stichwort „One Community, One Book" entwickelten Bibliothekar*innen Veranstaltungstypen, mit denen sich große Gruppen um einen Text versammeln konnten. Diese Formate differenzierten sich bald aus: Sie können unterschiedlich skaliert werden und damit die Bewohner eines Ortes oder einer Region, aber auch eines Unternehmens oder einer Institution umfassen. Zu beobachten sind auch Kooperationen von kommerziellen und nichtkommerziellen Akteuren und Motiven, die sich z. B. an Lese-Festivals oder TVBuchclubs beteiligen. Je nach Form der Kooperation sind zudem unterschiedliche Reichweiten und Rhythmen der so organisierten Leseprozesse vorstellbar, und natürlich unterschiedliche Kombinationen von virtuellen und physisch präsenten Leseanlässen 
und Lektürebegegnungen. Varianten solcher Veranstaltungen wurden auch bereits eingesetzt, um Erstsemester an ihrer Universität mit Hilfe eines gemeinsam gelesenen Textes in ihre Universität einzuführen. ${ }^{1}$

\section{Eine Uni - ein Buch}

Der Stifterverband der deutschen Wissenschaft und die Klaus Tschira Stiftung schreiben seit 2017 einen Wettbewerb aus, mit dem sie an die oben skizzierte Tradition anschließen. Unter dem Titel „Eine Uni - ein Buch“ können sich deutsche Hochschulen mit einem Leseprogramm über ein Semester bewerben. In bisher vier Jahren wurden jeweils zehn Teams mit 10.000 Euro ausgezeichnet. Insgesamt haben sich ca. 70 Hochschulen beworben. Die 40 ausgezeichneten Vorschläge werden auf der Webseite des Stifterverbands vorgestellt; dort sind auch die 3-minütigen Bewerbungsvideos der Ausgezeichneten abrufbar. ${ }^{2}$ Von den bisher angebotenen Titeln sind ca. 73 Prozent Sachbücher, ca. 26 Prozent stammen aus der Belletristik. Insgesamt wurden 14 Klassiker vorgeschlagen, was einem Anteil von ca. 19 Prozent entspricht. Unter den ausgezeichneten Bewerbungen verteilen sich die Gewichte etwas anders: Die Belletristik hat mit ca. 35 Prozent besser abgeschnitten, und der Anteil kanonischer Texte unter den Gewinnern liegt auch höher als in der Gesamtsumme der Einreichungen. Blickt man auf die Hochschultypen, so liegt der Anteil der Universitäten unter den Bewerbern bei ca. 53 Prozent; bei den Ausgezeichneten schneiden die Hochschulen für angewandte Wissenschaften mit ca. 52 Prozent etwas besser ab.

Weitere Einsichten kann man aus den Projektbeschreibungen und den BewerbungsVideos gewinnen, die allerdings auch Fragen aufwerfen: Welches Video wurde von einer Agentur geliefert, wo war die Kommunikationsabteilung zuständig, wo haben wir es mit Eigenproduktionen zu tun? Wie kam es überhaupt zur Bewerbung, und wie gestaltete sich der interne Auswahlprozess? Gleichwohl lassen die Filme einiges erkennen, weil sie in einer standardisierten Länge von 3 Minuten verdichten, wie man den mit dem Wettbewerb ausgesprochenen Leseauftrag interpretieren kann. Wie unterschiedlich dieser umgesetzt wurde, zeigt sich schon an zwei Fällen, in denen Kreativität als Thema gewählt wurde: Eine Hochschule verpflichtete sich darauf, nach der Kreativität im eigenen Arbeitsalltag zu suchen; für eine andere sollte das gemeinsam zu lesende Buch zur Herstellung eines erst noch zu produzierenden neuen Buchs führen. Hier umfasste die angestrebte Interaktion also Lesen und Schreiben.

Nicht wenige der Videos gleichen bewegten Power-Point-Präsentationen, in denen wir Menschen vor Bücherregalen sehen, die mit dem ausgewählten Buch in der Hand geplante Veranstaltungen beschreiben. Uni-Bibliotheken erscheinen häufig als Hintergrund, und in einigen ambitionierter gestalteten Videos wird auf die Bildidee des über die Schnitte weitergereichten Buches zurückgegriffen, um die Idee geteilten Wissens zu vermitteln. In anderen Bewerbungsfilmen schreibt man dem ausgewählten Buch ansatzweise einen Akteurs-Status in einem plot zu: Als roter Reclam-Band reist z. B. Huxleys Schöne neue Welt durch eine schwarz-weiß fotografierte dystopische Hochschulwelt, und in einem angedeuteten Krimi müssen sich zwei Kommissare mit einer mysteriösen Buchsendung befassen. In einem weiteren Film wird Camus' Die Pest(noch vor der Pandemie) in den Händen unterschiedlicher Lesender in gemütlichen 
Sesseln gezeigt und von kleinen Plüsch-Ratten als Leitmotiv begleitet. Insgesamt ist man bemüht, die Bewerbung als innovationsfreudig und technikaffin zu gestalten, so dass Visualisierungen von digitalen Endgeräten und Textoberflächen dominieren. Dies zeigt sich auch auf der Ebene der versprochenen Formate: In zumeist additiver Weise werden sowohl traditionelle Lesungen und Vorlesungen angeboten, als auch poetry slams, blogs, shared readings, discord servers und Reddit-Foren. In zwei Fällen wird angekündigt, das ausgewählte Buch der ganzen Hochschule in einer digitalisierten Version zugänglich zu machen, so dass es nicht in gedruckter Form beschafft werden müsse.

Die in den meisten Videos aufgefahrene Mischung von Lektüreformen und Lesetechnologien wird in den Bewerbungsfilmen so gut wie nie reflektiert. Tatsächlich werden das Lesen selbst, oder auch der Status des gedruckten Buches, nur in zwei von insgesamt 40 Bewerbungsfilmen überhaupt thematisiert. An einem Ort wählte man eine australische graphic novel ohne Worte und machte sich Gedanken darüber, wie der Rezeptionsprozess mehrsprachig gestaltet werden könnte. An einem anderen wurde die Materialität des gewählten Buchs in den Vordergrund gerückt und zur Hochschule in Beziehung gesetzt: Als anwendungsbezogene Einrichtung wollte man ein Interesse für das Gemachte und Gestaltbare, den Ding- und Werkcharakter von Büchern wecken. Überblickt man die Videos, so dominieren inhaltsbezogene Inszenierungen: Die meisten Bücher wurden mit Blick auf Themen ausgesucht, die an gegenwärtige gesellschaftliche Debatten anschließbar sind. Der Wettbewerbs-Auftrag scheint somit in der Mehrzahl der Fälle so interpretiert worden zu sein, dass mit Hilfe eines Buches ein möglichst resonantes Thema bestimmt wurde. Diese Priorisierung korreliert mit der dominanten Fokussierung auf Sachbücher: Eine Mehrheit der Bewerbungen schlägt Gegenwartsdiagnosen vor, die sich besonders gut als Anlässe zur Diskussion von aktuellen Herausforderungen eignen. Auch in Fällen, in denen auf Sachbuch-Klassiker wie Platons Der Staat oder Freiherr Knigges Über den Umgang mit Menschen zurückgegriffen wurde, legte man Wert darauf, dass die Klassizität des ausgewählten Textes seine Aktualisierbarkeit nicht ausschließt. Kein Bewerber-Team kam auf die Idee, die Auswahl seines Buches nicht mit seiner Kompatibilität für Gegenwartsdiskurse, sondern mit seiner historischen Alterität zu begründen - dies hat sicherlich auch damit zu tun, dass eine verständliche Sprache für die meisten Bewerbungen ein zentrales Auswahl-Kriterium war.

\section{Unity in Diversity}

Schon nach einer ersten Sichtung der Videos wird deutlich, dass die zentrale Herausforderung des Slogans „Eine Uni - ein Buch“ von so gut wie allen ausgezeichneten Teams im ersten Teil lokalisiert wird: Expliziert oder problematisiert werden weniger das Buch als Objekt noch das Lesen als buchgestützte Kulturtechnik, sondern die Tatsache, dass sich eine große und heterogene Gruppe um die Lektüre eines Textes versammeln soll. Diese Aufforderung zum gemeinsamen Lesen dient allen prämierten Bewerber-Gruppen als willkommene Gelegenheit, eine Bildsprache für das Ganze der jeweiligen Hochschule zu finden. Man erkennt schnell, dass in dieser Disziplin der institutionellen Selbstbeschreibung in den letzten Jahrzehnten hart trainiert 
wurde: Nicht wenige der Videos greifen auf strategische Bilder und Narrative, auf Leitbilder und mission statements zurück, um ihre Einrichtung nun als eine lesende zu präsentieren. In mindestens einem Fall wird im Bewerbungs-Video ganz explizit auf Verfahren zurückgegriffen, in denen es gilt, die eigene Hochschule möglichst kompetitiv zu singularisieren: Eine technische Universität trat mit dem Vorschlag an, Dürrenmatts Physiker dazu zu nutzen, die für die Exzellenz-Wettbewerbe entwickelte „Vision“ weiter zu entwickeln.

Die meisten prämierten Hochschulen haben das ausgewählte Buch somit als Mittel für andere Zwecke definiert: Wie in vielen Lesesituationen dient auch hier ein Text als Anlass, um sich - im doppelten Sinn - zu unterhalten, Lösungen zu entwickeln und an der Organisationsentwicklung zu arbeiten. Ein solches strategic reading hat es im Fall der Hochschulentwicklung mit spezifischen Herausforderungen zu tun: Hier gilt es, auch die kollektiv organisierte Lektüre dazu zu nutzen, um einen Haufen individualistisch gesinnter Einzeltäter*innen und Einzelleser*innen auf eine Vorstellung von teilbaren Werten und Zielen einzustimmen. Im Wettbewerb „Eine Uni - ein Buch“ verdichten sich daher noch einmal jene Fragen, um die es auch in den Exzellenz-Wettbewerben geht: Wie werden Hochschulen als kollektive Akteure handlungs- und wettbewerbsfähiger; und hilft es ihnen dabei, sich Maßnahmen aus der Unternehmenswelt anzueignen? Liest eine Uni besonders dann bereitwillig und anregend ein Buch, wenn sie schon eine corporate identityausgebildet hat?

Die Videos der ausgezeichneten Hochschulen bieten zu diesen Fragen Anschauungsmaterial. Man hat den Eindruck, dass die Gestaltungsenergie vor allem für die Frage mobilisiert wurde, wie die Teile und das Ganze der Hochschule möglichst überzeugend als verbunden dargestellt werden können. Die Stilmittel, die zur Bewältigung dieser Aufgabe eingesetzt werden, ähneln sich: Das Leitmotiv nicht nur der Organisationsentwicklung, sondern auch der gemeinsamen Lektüre scheint unity in diversity zu sein. Fast allen Filmen gelingt es auf zeitgemäße Weise, Akteur*innen aus verschiedenen Bereichen der jeweiligen Hochschule und vor allem mit unterschiedlichen Positionen und kulturellen Hintergründen so zu versammeln, dass der Eindruck eines lebendigen und vielstimmigen Ganzen entsteht, in dem an einer gemeinsamen Sache gearbeitet wird. Gleichstellungsstandards werden offensichtlich in der Gestaltung solcher Wettbewerbsbeiträge insoweit berücksichtigt, dass viele Filme mit jungen Frauen einsetzen und diese als Kommentatorinnen eine Rolle spielen lassen. Wo die Hochschulleitung ins Bild kommt, werden weibliche Führungskräfte mobilisiert.

Studierende spielen in den meisten Fällen eine sichtbare und hörbare Rolle und strahlen Mitgestaltungsmacht aus. Insgesamt entsteht beim Blick auf die Darstellung des Ganzen im Brennglas eines einzigen Buches der Eindruck, dass es weniger um die Integration von Lesehaltungen und -erfahrungen geht, sondern um die Vermittlung von integrierter sozialer Diversität mit Hilfe eines thematisch passförmigen Textes.

Was sich in den vierzig Videos also zeigt, sind institutionelle Stile der Repräsentativität als Vorgriff auf ein Ganzes, in dem sich möglichst viele Mitglieder berücksichtigt, vertreten und anerkannt fühlen. Ob dieses im jeweiligen Fall bereits existiert oder noch entstehen muss, ist aus den Filmen nicht eindeutig abzuleiten. Aufschlussreich sind 
aber unterschiedliche Umgänge mit Status-Unterschieden: In den meisten Fällen wurde nicht darauf verzichtet, die auftretenden Personen zu identifizieren. Hier wäre noch zu fragen, wie die jeweils gewählten Strategien der institutionellen Selbstdarstellung mit Hilfe eines Buches zu deuten sind: Hat die technische Universität, die sich mit Asterix beschäftigen möchte, einfach nur ehrlich-realistisch agiert, indem sie erst einmal sechs Männer aus technischen Disziplinen auf den Laufsteg ihres Videos schickte? Besonders deutlich unterscheidet sich von dieser Bewerbung der Film der Universität, die Knigges Vom Umgang mit den Menschen bestimmt hat. Sie wählte eine riskantere ironische Option: Die Personen, die hier auftreten, sind nicht benannt. Die wiederkehrende Figur eines mittelalten Mannes agiert dezidiert verlegen und unterminiert den Habitus männlichen Expertentums, während mehrere Frauen sich durch Gewandtheit und Eloquenz auszeichnen. Die Selbstinszenierung der Universität in diesem Film liefert somit einen indirekten Kommentar zum Thema ihres Buches, in dem die Macht von symbolischen Formen zur Bewältigung sozialer Interaktion im Mittelpunkt steht.

Die Beiträge zu „Eine Uni - ein Buch“ beleuchten das gegenwärtige Bedürfnis nach gemeinschaftsstiftenden Lektürepraktiken und -formaten in einem akademischen Kontext. Sie können mit aktuellen Themen am KWI in Verbindung gebracht werden: Mit einem Workshop zu geteilten Autor*innenschaften und pluralisierten Werken und einer Diskussion mit Heinz Bude, Bettina Munk und Karin Wieland, den Autor*innen des Romans Aufprall, in der sich kollektive Lese- und Schreiberfahrungen verbinden werden. Und natürlich könnten auch wir uns die Frage stellen, welches Buch wir nicht nur in kleineren Lesegruppen, sondern als institutionelles Ganzes auswählen, und auf welche Weisen wir es lesen würden?

\section{References}

1. Ein guter Überblick über neuere Organisationsformen kollektiven Lesens und eine Reihe von empirischen Fallstudien finden sich in Danielle Fuller, DeNel Rehberg Sedo. Reading Beyond the Book. The Social Practices of Contemporary Literary Culture. New York, London: Routledge, 2013.

2. Vgl. https://www.stifterverband.org/eine-uni-ein-buch. Für weitere Hinweise danke ich Volker Meyer-Guckel, dem stellvertretenden Generalsekretär des Stifterverbands.

SUGGESTED CITATION: Griem, Julika: Gemeinsam lesen?, in: KWI BLOG, [https://blog.kulturwissenschaften.de/gemeinsam-lesen/], 08.03.2021

DOI: https://doi.org/10.37189/kwi-blog/20210308-0830 
Dieser Text wird über DuEPublico, dem Dokumenten- und Publikationsserver der Universität Duisburg-Essen, zur Verfügung gestellt. Die hier veröffentlichte Version der EPublikation kann von einer eventuell ebenfalls veröffentlichten Verlagsversion abweichen.

DOI: $\quad 10.37189 / \mathrm{kwi}-\mathrm{blog} / 20210308-0830$

URN: urn:nbn:de:hbz:464-20210308-090206-2 